University of Texas at El Paso

ScholarWorks@UTEP

8-2005

\title{
Discrete Conservation of Nonnegativity for Elliptic Problems Solved by the hp-FEM
}

Pavel Solin

Thomas Vejchodsky

Roberto Araiza

The University of Texas at El Paso, raraiza@miners.utep.edu

Follow this and additional works at: https://scholarworks.utep.edu/cs_techrep

Part of the Computer Engineering Commons

Comments:

UTEP-CS-05-29.

Published in Mathematics and Computers in Simulation, 2007, Vol. 76, pp. 205-210.

\section{Recommended Citation}

Solin, Pavel; Vejchodsky, Thomas; and Araiza, Roberto, "Discrete Conservation of Nonnegativity for Elliptic Problems Solved by the hp-FEM" (2005). Departmental Technical Reports (CS). 261.

https://scholarworks.utep.edu/cs_techrep/261

This Article is brought to you for free and open access by the Computer Science at ScholarWorks@UTEP. It has been accepted for inclusion in Departmental Technical Reports (CS) by an authorized administrator of ScholarWorks@UTEP.For more information, please contact Iweber@utep.edu. 


\title{
Discrete Conservation of Nonnegativity for Elliptic Problems Solved by the $h p$-FEM
}

\author{
Pavel Šolín ${ }^{1}$ \\ Academy of Sciences of the Czech Republic, Prague \\ University of Texas at El Paso, El Paso, TX \\ Tomáš Vejchodský² \\ Academy of Sciences of the Czech Republic, Prague \\ Roberto Araiza \\ University of Texas at El Paso, El Paso, TX
}

\begin{abstract}
Most results related to discrete nonnegativity conservation principles (DNCP) for elliptic problems are limited to finite differences (FDM) and lowest-order finite element methods (FEM). In this paper we confirm that a straightforward extension to higher-order finite element methods ( $h p$ FEM) in the classical sense is not possible. We formulate a weaker DNCP for the Poisson equation in one spatial dimension and prove it using an interval computing technique. Numerical experiments related to the extension of this result to $2 \mathrm{D}$ are presented.
\end{abstract}

Keywords: discrete nonnegativity conservation, elliptic problems, $h p$-FEM

\section{Introduction}

Numerical methods for partial differential equations (PDEs) are used extensively in various areas of engineering and science. When a PDE contains a nonnegative quantity such as, e.g., the density, pressure, temperature, or concentration, then the numerical method is naturally expected to deliver its nonnegative approximation. However, it is not obvious why this should be the case, and as a matter of fact, sometimes the approximations of nonnegative quantities come out negative.

The explanation of this occasional nonphysical behavior is that numerical schemes such as the finite element or finite difference methods usually are not born with mechanisms for detection of physically wrong solutions. Usually a deep analysis of the numerical method is needed to determine conditions under which the approximation of nonnegative physical quantities will be nonnegative. Results of this type are called discrete nonnegativity conservation principles

\footnotetext{
${ }^{1}$ The first author acknowledges the financial support of the Grant Agency of the Czech Republic under the Project No. 102/05/0629.

${ }^{2}$ The work of the second author was partially supported by the Grant Agency of the Czech Republic under the Project No. 201/04/P021 and by the Academy of Sciences of the Czech Republic Institutional Research Plan No. AV0Z10190503.
} 
(DNCP), and their formulation is highly nontrivial even for the simplest PDEs discretized by the simplest numerical methods $[1,2]$.

DNCP often bring additional restrictions on problem data or parameters of the numerical method. They are well known for various types of lowest-order methods, such as for piecewise-affine FEM (see, e.g., $[5,6,7,11,12]$ )). Because of the complexity of higher-order finite element methods (see, e.g., [9] and the references therein), virtually no results are available for the $p$ - and $h p$-FEM.

There was one exception, perhaps: In early 1980s Höhn and Mittelmann [3] formulated a DNCP for quadratic Lagrange elements in 2D and proved that it did not work but under prohibitive restrictions on the shape of the triangulation.

\section{A One-Dimensional Example}

Höhn and Mittelmann [3] tried to extend to higher-order elements a classical DNCP which was known to work in the piecewise-affine case. Let us present a simple model problem showing that even in one spatial dimension such strategy does not have a chance to be successful:

Consider the Poisson equation

$$
-\Delta u=f
$$

with homogeneous Dirichlet boundary conditions and a nonnegative right-hand side

$$
f(x)=200 \mathrm{e}^{-10(x+1)}
$$

in the interval $\Omega=(-1,1)$. According to a standard maximum principle for elliptic problems, the exact solution $u$ is nonnegative in the entire domain $\Omega$. The finite element formulation of problem (2.1) reads:

Find $u_{h, p} \in V_{h, p}$ such that

$$
\int_{\Omega} u_{h, p}^{\prime}(x) v_{h, p}^{\prime}(x) \mathrm{d} x=\int_{\Omega} f(x) v_{h, p}(x) \mathrm{d} x \quad \text { for all } v_{h, p} \in V_{h, p},
$$

where $V_{h, p}$ is a piecewise-polynomial space determined by the finite element mesh.

Let $\Omega$ be covered with a single finite element $K_{1}=(-1,1)$. When this element is equipped with the polynomial degree $p\left(K_{1}\right)=1$, then $V_{h, p}=\{0\}$ and the approximate solution $u_{h, p}$ is the zero function. This function is nonnegative and therefore the DNCP holds. However, when we choose $p\left(K_{1}\right)=3$, then $V_{h, p}=P_{0}^{3}(-1,1)$ and the finite element solution

$$
u_{h, p}(x)=\frac{1}{40}\left[54+66 \mathrm{e}^{-20}-\left(73-133 \mathrm{e}^{-20}\right) x\right]\left(1-x^{2}\right)
$$

is negative in a subset of $\Omega$ (evaluate, e.g., at $x=0.9$ ). 


\section{Stronger Assumptions on the RHS}

Thus the standard assumption of nonnegativity of the right-hand side $f$, which was sufficient in the piecewise-affine FEM [2], is not enough already for cubic elements in one spatial dimension.

Let us understand what happened: Let $V_{h, p}=P_{0}^{p}(-1,1)$, where $p=3$, be the space of cubic polynomials vanishing at $\Omega$-endpoints. Consider the $L^{2}$ projection $f_{h, p}$ of the function $f$ to the space $W_{h, p}=P^{p}(-1,1)$ of cubic polynomials,

$$
\int_{\Omega}\left[f(x)-f_{h, p}(x)\right] v_{h, p}(x) \mathrm{d} x=0 \quad \text { for all } v_{h, p} \in W_{h, p} .
$$

Since $V_{h, p} \subset W_{h, p}$, it follows from (3.5) that problem (2.3) is equivalent to the problem

$$
\int_{\Omega} u_{h, p}^{\prime}(x) v_{h, p}^{\prime}(x) \mathrm{d} x=\int_{\Omega} f_{h, p}(x) v_{h, p}(x) \mathrm{d} x \quad \text { for all } v_{h, p} \in V_{h, p},
$$

where the original right-hand side $f$ was replaced with its $L^{2}$-projection $f_{h, p}$.

It is easy to calculate from (3.5) that

$$
f_{h, p}=-8.25+29.175 x+54.75 x^{2}-93.625 x^{3} .
$$

This function is negative in a subset of $\Omega$ (evaluate, e.g., at $x=0$ ).

In other words, although the original right-hand side $f$ was nonnegative, the contributions to the load vector were equivalent to those produced by a different function $f_{h, p}$ which was negative in a subset of $\Omega$. This is the reason why the cubic approximation (2.4) was negative in a subset of $\Omega$. This observation motivates the following theorem:

Theorem 3.1 Let $\Omega=(-1,1)$. Consider the discrete problem (2.3) on a single finite element $K_{1}=(-1,1)$ of the polynomial degree $p\left(K_{1}\right)>1$. Let the righthand side $f \in L^{2}(\Omega)$ be such that its $L^{2}$-projection to the polynomial space $W_{h, p}=P^{p}(\Omega)$, defined by $(3.5)$, is nonnegative in $\Omega$. Then the solution $u_{h, p}$ of the discrete problem (2.3) is nonnegative.

\section{Idea of Proof}

The proof of Theorem 3.1 uses the Lobatto shape functions (see, e.g., [8]),

$$
l_{k}(x)=\frac{1}{\left\|L_{k-1}\right\|_{L^{2}}} \int_{-1}^{x} L_{k-1}(\xi) \mathrm{d} \xi, \quad 2 \leq k .
$$

Here $L_{0}, L_{1}, \ldots$ are the Legendre polynomials with $\left\|L_{k-1}\right\|_{L^{2}}=\sqrt{2 /(2 k-1)}$. It follows from (4.7) that the functions $l_{2}, l_{3}, \ldots$ vanish for $x=-1$ and $x=1$, and moreover that they are orthonormal in the $H_{0}^{1}(-1,1)$ inner product,

$$
\left(l_{i}, l_{j}\right)_{H_{0}^{1}(-1,1)}=\int_{-1}^{1} l_{i}^{\prime}(x) l_{j}^{\prime}(x) \mathrm{d} x=\delta_{i j}, \quad 2 \leq i, j .
$$


The functions $l_{2}, l_{3}, \ldots, l_{p}$ can be used as a basis in the space $V_{h, p}=P_{0}^{p}(\Omega)$ and the approximate solution $u_{h, p} \in V_{h, p}$ can be expressed in the standard form

$$
u_{h, p}(x)=\sum_{i=1}^{p-1} y_{i} l_{i+1}(x) .
$$

Substituting (4.8) into (2.3) and using the functions $l_{2}, l_{3}, \ldots, l_{p}$ as test functions, we find that the unknown coefficients $y_{1}, y_{2}, \ldots, y_{p-1}$ satisfy

$$
y_{i}=\int_{-1}^{1} \sum_{j=1}^{p-1} y_{j} l_{j+1}^{\prime}(x) l_{i+1}^{\prime}(x) \mathrm{d} x=\int_{-1}^{1} f_{h, p}(x) l_{i+1}(x) \mathrm{d} x, \quad 1 \leq i \leq p-1 .
$$

Putting (4.10) back to (4.9), we obtain

$$
u_{h, p}(x)=\sum_{i=1}^{p-1}\left(\int_{-1}^{1} f_{h, p}(z) l_{i+1}(z) \mathrm{d} z\right) l_{i+1}(x)=\int_{-1}^{1} f_{h, p}(z) \Phi_{p}(x, z) \mathrm{d} z,
$$

where

$$
\Phi_{p}(x, z)=\sum_{i=1}^{p-1} l_{i+1}(x) l_{i+1}(z) .
$$

For every $p>1$, the function $\Phi_{p}(x, z)$ is a given bivariate polynomial defined in the square $(-1,1)^{2}$. It is our goal to show that $u_{h, p}(x)$ is nonnegative for all $x \in(-1,1)$ using $(4.11)$. This is done for each polynomial degree $p$ separately in two steps:

1. Identify a subdomain $\Omega_{p}^{+}$of $(-1,1)$ where the function $\Phi_{p}$ is positive.

2 . Find a quadrature rule of the order of accuracy $2 p$ (exact for all polynomials of degree less or equal to $2 p$ ) with positive weights and points lying in $\Omega_{p}^{+}$.

The construction of the subdomains $\Omega_{p}^{+}$and the corresponding quadrature rules finishes the proof. The concrete subdomains $\Omega_{p}^{+}$along with the quadrature rules can be found in [10]. The interval computation technique based on exact integer arithmetics, which was used to verify that the functions $\Phi_{p}$ were positive in the subdomains $\Omega_{p}^{+}$, is described in Section 5 .

\section{Application of Interval Arithmetics}

Since the presented proof is computer-aided, one has to be especially careful to avoid doubts concerning the finite arithmetics, rounding errors, etc. Let us demonstrate the procedure on the quartic case, where we deal with the function

$$
\Phi_{4}(x, z)=\sum_{i=1}^{3} l_{i+1}(x) l_{i+1}(z)=\left(x^{2}-1\right)\left(z^{2}-1\right) \Psi_{4}(x, z),
$$


where

$$
\Psi_{4}(x, z)=\frac{3}{8}+\frac{5}{8} x z+\frac{7}{128}\left(5 x^{2}-1\right)\left(5 z^{2}-1\right) .
$$

The function $\Phi_{4}(x, z)$ is shown in Fig. 1 .

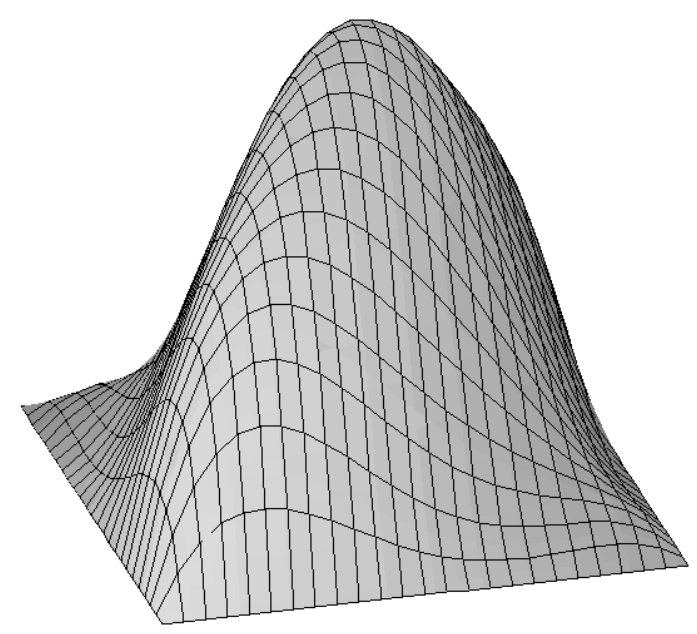

Figure 1: The function $\Phi_{4}(x, z)$.

If $\Phi_{4}(x, z)$ really is nonnegative in the entire square $[-1,1]^{2}$ as Fig. 1 suggests, then by (4.11) the quartic approximation $u_{h, p} \in P_{0}^{4}(-1,1)$ is nonnegative and the proof for $p=4$ finished. We prove its nonnegativity using integer arithmetics via the interval computing technique [4].

In interval computing one deals with intervals instead of numbers, and standard unary and binary operations are extended from numbers to intervals in a natural way. For example, $[\underline{a}, \bar{a}]+[\underline{b}, \bar{b}]=[\underline{a}+\underline{b}, \bar{a}+\bar{b}],[\underline{a}, \bar{a}]-[\underline{b}, \bar{b}]=[\underline{a}-\bar{b}, \bar{a}-\underline{b}]$, and so on. If we replace every operation with numbers by the corresponding operation of interval arithmetic, we get an enclosure for the range of the analyzed function on given intervals [4].

Let us use this technique to prove the nonnegativity of the function (5.12) in the square $[-1,1]^{2}$ : Substituting a pair of intervals $X=[\underline{x}, \bar{x}]$ and $Z=[\underline{z}, \bar{z}]$ into (5.12), we obtain an enclosure

$$
\left[\underline{\Psi}_{4}, \bar{\Psi}_{4}\right] \supseteq \Psi_{4}(X, Z)=\left\{\Psi_{4}(x, z) ; x \in X, z \in Z\right\} .
$$

Moreover, since the function (5.12) is polynomial and it only contains rational coefficients, its evaluation for rational intervals can be done using exact integer arithmetic.

Step 1: Consider the intervals $X_{1}=Z_{1}=[-1,1]$, and compute the enclosure $\left[\underline{\Psi}_{4}, \bar{\Psi}_{4}\right]$ for $\Psi_{4}\left(X_{1}, Z_{1}\right)$ :

$$
\left[\underline{\Psi}_{4}, \bar{\Psi}_{4}\right]=[-25 / 16,95 / 32] \supseteq \Psi_{4}\left(X_{1}, Z_{1}\right) .
$$


If the left endpoint $\underline{\Psi}_{4}$ of the enclosure interval $\left[\underline{\Psi}_{4}, \bar{\Psi}_{4}\right]$ was nonnegative, then the proof would be finished. Since this is not the case, we refine the grid by halving both the intervals $X_{1}$ and $Z_{1}$. We obtain four subdomains $[-1,0] \times[-1,0]$, $[-1,0] \times[0,1],[0,1] \times[-1,0]$, and $[0,1] \times[0,1]$.

Step 2: Compute the enclosures for these subdomains:

- for $[-1,0] \times[-1,0]$, we get $\left[\underline{\Psi}_{4}, \bar{\Psi}_{4}\right]=[5 / 32,15 / 8] \supseteq \Psi_{4}([-1,0],[-1,0])$;

- for $[-1,0] \times[0,1]$, we get $\left[\underline{\Psi}_{4}, \bar{\Psi}_{4}\right]=[-15 / 32,5 / 4] \supseteq \Psi_{4}([-1,0],[0,1])$;

- for $[0,1] \times[-1,0]$, we get $\left[\underline{\Psi}_{4}, \bar{\Psi}_{4}\right]=[-15 / 32,5 / 4] \supseteq \Psi_{4}([0,1],[-1,0])$;

- for $[0,1] \times[0,1]$, we get $\left[\underline{\Psi}_{4}, \bar{\Psi}_{4}\right]=[5 / 32,15 / 8] \supseteq \Psi_{4}([0,1],[0,1])$.

This proves that the function $\Psi_{4}$ (and hence also $\Phi_{4}$ ) is nonnegative in the subdomains $[-1,0] \times[-1,0]$ and $[0,1] \times[0,1]$. As for the remaining subdomains $[-1,0] \times[0,1]$ and $[0,1] \times[-1,0]$, we divide each of them into four equal subdomains, compute the enclosure for each new subdomain, etc.

After five iterations of this procedure, we get a partition of $[-1,1]^{2}$ for which the left endpoints of the enclosures are nonnegative. So we have proved that $\Psi_{4}$ (and hence also $\Phi_{4}$ ) is nonnegative in $[-1,1]^{2}$. Both the Java programs and output files with details on the computations for $p=4,5, \ldots, 10$ can be viewed on the web page http://www. math.utep.edu/Faculty/solin/intcomp.

\section{A Two-Dimensional Example}

From the one-dimensional example shown in Section 2 it is clear that an extension of the DNCP from the lowest-order FEM to the $h p$-FEM only is possible in some weaker form. Most likely, one will have to impose suitable restrictions on the $L^{2}$-projection of the right-hand side, analogously to the one-dimensional case. However, such conditions are an open problem, and it turns out that they have to be even stronger than in the one-dimensional case. This hypothesis is motivated by the following numerical experiment:

Consider the Poisson equation $-\Delta u=f$ with a nonnegative cubic polynomial right-hand side $f\left(x_{1}, x_{2}\right)=-1000\left(x_{1}+x_{2}-2\right)^{3}$ in a square domain $\Omega=(-1,1)^{2}$. The problem is equipped with homogeneous Dirichlet boundary conditions, and we will solve it using two different meshes consisting of two cubic triangular elements $K_{1}, K_{2}$ :

(A) $K_{1}=([-1,-1],[1,-1],[-1,1])$ and $K_{2}=([1,-1],[1,1],[-1,1])$,

(B) $K_{1}=([-1,-1],[1,1],[-1,1])$ and $K_{2}=([-1,-1],[1,-1],[1,1])$.

The approximate solution corresponding to the mesh $(\mathrm{A})$ is nonnegative in the entire domain $\Omega$, as shown in Fig. 2 . 


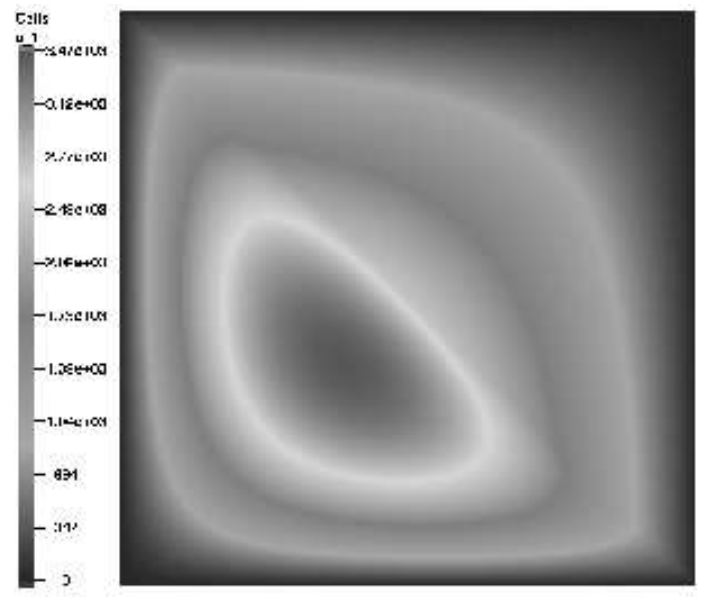

Figure 2: Nonnegative piecewise cubic solution corresponding to the mesh (A).

However, the approximation obtained on the mesh (B), shown in Fig. 3, is negative in a subset of $\Omega$.

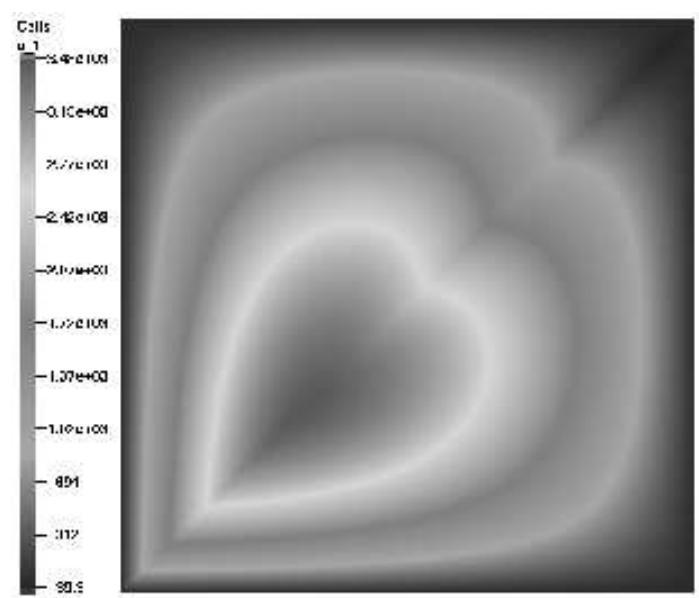

Figure 3: The piecewise cubic solution corresponding to the mesh (B) is negative close to the upper-right corner of $\Omega$.

It follows from this example that an assumption of nonnegativity of the $L^{2}$ projection of the right-hand side, which was sufficient in one dimension, is not enough already on triangular elements in two dimensions. The investigation of this phenomenon is one of our current research goals. 


\section{References}

[1] P.G. Ciarlet: Discrete Maximum Principle for Finite Difference Operators, Aequationes Math. 4, pp. 338 - 352, 1970.

[2] P.G. Ciarlet, P.A. Raviart: Maximum principle and uniform convergence for the finite element method, Computer Methods Appl. Mech. Engrg. 2, pp. $17-31,1973$.

[3] W. Höhn, H.D. Mittelmann: Some Remarks on the Discrete Maximum Principle for Finite Elements of Higher-Order, Computing 27, pp. 145 154, 1981.

[4] L. Jaulin, M. Kieffer, O. Didrit, E. Walter: Applied interval analysis, Springer Verlag, London, 2001.

[5] J. Karátson, S. Korotov: Discrete maximum principles for finite element solutions of nonlinear elliptic problems with mixed boundary conditions, Numer. Math. 99, pp. $669-698,2005$.

[6] S. Korotov, M. Křřžek, P. Neittaanmäki: Weakened acute type condition for tetrahedral triangulations and the discrete maximum principle, Math. Comp. 70, pp. 107 - 119, 2000.

[7] M. Kř́̌žek, L. Liu: On the maximum and comparison principles for a steady-state nonlinear heat conduction problem, ZAMM Z. Angew. Math. Mech. 83, pp. $559-563,2003$.

[8] P. Solín: Partial Differential Equations and the Finite Element Method, J. Wiley \& Sons, 2005.

[9] P. Šolín, K. Segeth, I. Doležel: Higher-Order Finite Element Methods, Chapman \& Hall/CRC Press, Boca Raton, 2003.

[10] P. Šolín, T. Vejchodský: On the Discrete Maximum Principle for the $h p$ FEM, submitted.

[11] T. Vejchodský: On the Nonnegativity Conservation in Semidiscrete Parabolic Problems. In: M. Křŕžek, P. Neittaanmäki, R. Glowinski, S. Korotov (Eds.), Conjugate Gradients Algorithms and Finite Element Methods, Berlin, Springer-Verlag 2004, pp. 197-210.

[12] T. Vejchodský: Method of Lines and Conservation of Nonnegativity. In: Proc. of the European Congress on Computational Methods in Applied Sciences and Engineering (ECCOMAS 2004), Jyväskylä, Finland, 2004. 